RESEARCH

\title{
THE PSOAS MUSCLE INDEX AND RETROSPECTIVE CLINICAL FRAILTY SCALE SCORE PREDICT OVERALL SURVIVAL IN PATIENTS WITH MULTIPLE MYELOMA
}

Turkish Journal of Geriatrics DOI: 10.31086/tigeri.2021.249 2021; 24(4): 518-525

- Mahmut Bakır KOYUNCU1

- Tolga KÖSECi2

- Erkan GÜLER ${ }^{3}$

- Barış TEN ${ }^{4}$

- Feramuz Demir APAYDIN ${ }^{4}$

- Erdinç NAYIR ${ }^{5}$

- Hakan BASIR 6

- Aydan AKDENIZ 6

- AnII TOMBAK ${ }^{6}$

- Eyüp Naci TiFTiK 6

\section{CORRESPONDANCE}

\section{${ }^{1}$ Mahmut Bakır KOYUNCU}

Adana City Research and Training Hospital, Hematology, Adana, Turkey

Phone: +9055535338853

e-mail: mahmutbakirkoyuncu@gmail.com

Received: Jul 05, 2021

Accepted: Nov 10, 2021

1 Adana City Research and Training Hospital, Hematology, Adana, Turkey

${ }^{2}$ Adana City Research and Training Hospital, Medical Oncology, Adana, Turkey

${ }^{3}$ Mersin University Faculty of Medicine, General Surgery, Mersin, Turkey

${ }^{4}$ Mersin University Faculty of Medicine, Radiology, Mersin, Turkey

${ }^{5}$ VM Medical Park Hospital, Medical Oncology, Mersin, Turkey

${ }^{6}$ Mersin University Faculty of Medicine, Hematology, Mersin, Turkey

\section{Abstract}

Introduction: Using the retrospective Clinical Frailty Scale (CFS) to assess frailty and the psoas muscle index (PMI) to determine sarcopenia status are new concepts. Since the cut-off values of the PMI differ by race, populationspecific cut-offs are recommended. We aimed to investigate the prognostic significance of retrospective CFS scores and sarcopenia status determined by population-specific PMl cut-offs in patients with multiple myeloma (MM).

Materials and Method: We included 111 patients who met the inclusion criteria. The patients' PMIs were calculated by measuring the psoas muscle at the level of the L3 vertebra on CT images. The CFS scores of the patients were retrospectively determined.

Results: While $46(41.4 \%)$ of the patients had sarcopenia, 79 (72\%) were frail. The median follow-up duration was 21.7 (1.51-74.97) months. The median overall survival was 42.3 months. The risk of death was significantly higher in patients with sarcopenia than in patients without sarcopenia (log-rank $p=0.028$, HR: $2.43,95 \% \mathrm{Cl}: 1.02-5.77)$, and frailty significantly affected overall survival (HR: $3.28,95 \% \mathrm{Cl}: 1.28-8.41, \mathrm{p}=0.01$ ).

Conclusion: Sarcopenia is a common finding in patients with MM. Both retrospective CFS scores and sarcopenia status, as determined by the PMI, were significantly associated with overall survival in patients with MM.

Keywords: Multiple Myeloma; Sarcopenia; Frailty. 


\section{INTRODUCTION}

Multiple myeloma (MM) is a malignant disease characterized by increased plasma cells in the bone marrow. The spectrum of this disease is broad. Patients with MM may present with many findings, such as anemia, hypercalcemia, renal disease, fatigue, weight loss, and bone pain[1]. The concepts of frailty and sarcopenia are essential in determining prognosis and treatment strategies and predicting treatment complications for patients with solid tumors[2,3]. Recent studies have shown that frailty is also crucial in determining the prognosis of patients with MM[4]. However, few studies have evaluated the effects of sarcopenia in these patients.

Sarcopenia refers to decreased muscle size and function. It is associated with mortality, disability, falls, a decline in physiological reserve, and low quality of life, especially in the elderly population. Recent studies on solid tumors have shown that low skeletal muscle mass is correlated with worse clinical outcomes regardless of age[5, 6]. According to the European Working Group on Sarcopenia in Older People (EWGSOP2) algorithm, CT (Computed Tomography) is the gold standard to verify sarcopenia[7]. CT-based muscle measurements can be efficient when CT is used routinely for diagnosis and follow-up, such as in patients with malignant diseases. Measuring the total cross-sectional muscle area from the L3 vertebra alignment, as defined by Prado et al., is associated with overall survival and adverse outcomes, and was also found to be highly correlated with total body muscle mass[6]. Subsequently, studies have shown that measuring only the psoas muscle area rather than the entire muscle area is quite simple and valuable in some cases[8,9]. The cut-off values for psoas muscle measurements remain unclear and vary by race. Thus, the EWGSOP2 recommended this topic as a high-priority study area. A study conducted by Bahat et al. demonstrated that the psoas muscle index (PMI) cut-off values in the Turkish population differed from the cut-off values recommended by Prado et al.[10].
Frailty is a syndrome that involves a general decrease in body functions. It is well known that frailty is a cause of sarcopenia, especially in elderly patients. The most widely used tool for frailty screening is the Clinical Frailty Scale (CFS). In this scoring system, patients are given one point (very fit) to nine points (terminally ill). The CFS is a reliable frailty screening tool in the Turkish population[11]. A validation study emphasized that retrospective use of the CFS is highly reliable[12]. Although many studies in the literature have shown that clinical frailty scores help predict prognosis, overall survival, and treatment toxicities in $\mathrm{MM}$, there is not yet a study on the use of a retrospective CFS in patients with $\operatorname{MM}[13,14]$.

This study aimed to determine the relationships among sarcopenia, frailty and overall survival in patients with MM.

\section{MATERIALS AND METHOD}

\section{Sample and study design}

Among the 157 patients diagnosed with MM at the Mersin University Department of Hematology between 2015 and 2020, we included 111 patients. The inclusion criteria were as follows: patients who underwent PET/CT for bone surveys at the time of diagnosis and were started on the Cybord chemotherapy protocol (weekly bortezomib, cyclophosphamide, and dexamethasone). Demographic characteristics, the initial evaluation of MM, the genetic risk category, and survival after diagnosis were recorded. We excluded patients who underwent an imaging method other than PET/CT at the time of diagnosis (24 patients), patients who did not accept treatment (11 patients), patients who had received a chemotherapy protocol other than Cybord (9 patients), and patients with poor image quality at the level of the L3 vertebra (2 patients).

\section{Frailty assessment}

Rockwood et al. developed the CFS to determine frailty status according to patients' cognition, 
physical examinations, comorbidities, and disabilities[15]. The CFS scores of the patients were retrospectively determined, based on a retrospective CFS validation study by Stille et al.[12]. CFS scores range from one to nine points (Level-1: very fit, Level-2: fit, Level-3: managing well, Level-4: living with very mild frailty, Level-5: living with mild frailty, Level-6: living with moderate frailty, Level-7: living with severe frailty, Level-8: living with very severe frailty, Level-9: terminally ill). The validity and reliability of this scale were previously reported in the Turkish population[11]. Each point on this scale corresponds to a written description of frailty, complemented by a visual chart to assist with frailty classification. CFS scores were calculated for each patient based on clinical judgment. Patients who scored 1-3 were classified as normal; patients who scored 4 were scored as vulnerable; and patients who scored $\geq 5$ were classified as frail. In our study, the patients were divided into two groups based on their frailty status (frail for scores $>3$ and robust for scores $\leq 3$ ).

\section{CT image analysis and sarcopenia assessment}

The right and left psoas muscle borders were manually drawn by co-author FDA (a board-certified radiologist) on CT images at the level of the L3 vertebra. The images were analyzed using ExtremePACS version 3.4 (Hacettepe Teknokent, Turkey). The left psoas area (LPA) and right psoas area (RPA) values were obtained in $\mathrm{mm}^{2}$. The average densities of the right and left psoas muscle were measured in Hounsfield units (HU) (Figure 1).

The PMI was calculated in $\mathrm{mm}^{2} / \mathrm{m}^{2}$ using the following equation: $\mathrm{PMI}=(\mathrm{RPA}+\mathrm{LPA}) /$ height $^{2}$. The Hounsfield unit average calculation (HUAC) was obtained using the formula [(right mean psoas density $\times$ RPA) + (left mean psoas density $\times$ LPA)] / (RPA + LPA). A PMI $<540 \mathrm{~mm}^{2} / \mathrm{m}^{2}$ for men and $<360 \mathrm{~mm}^{2} /$ $\mathrm{m}^{2}$ for women were the cut-off points to define sarcopenia according to a Turkish population study[10].

\section{Ethical approval}

This study was conducted in compliance with the principles of the Declaration of Helsinki. It was approved by the Mersin University Faculty of Medicine's Ethics Committee (Decision date: 14.10.2020 Number:2020/708).

\section{Statistical analysis}

Data distribution characteristics were evaluated using the Kolmogorov-Smirnov test, skewness and kurtosis assessment, coefficients of variation,

Figure 1. Demonstration of the left and right psoas muscle borders at the level of the L3 vertebra (CT image)

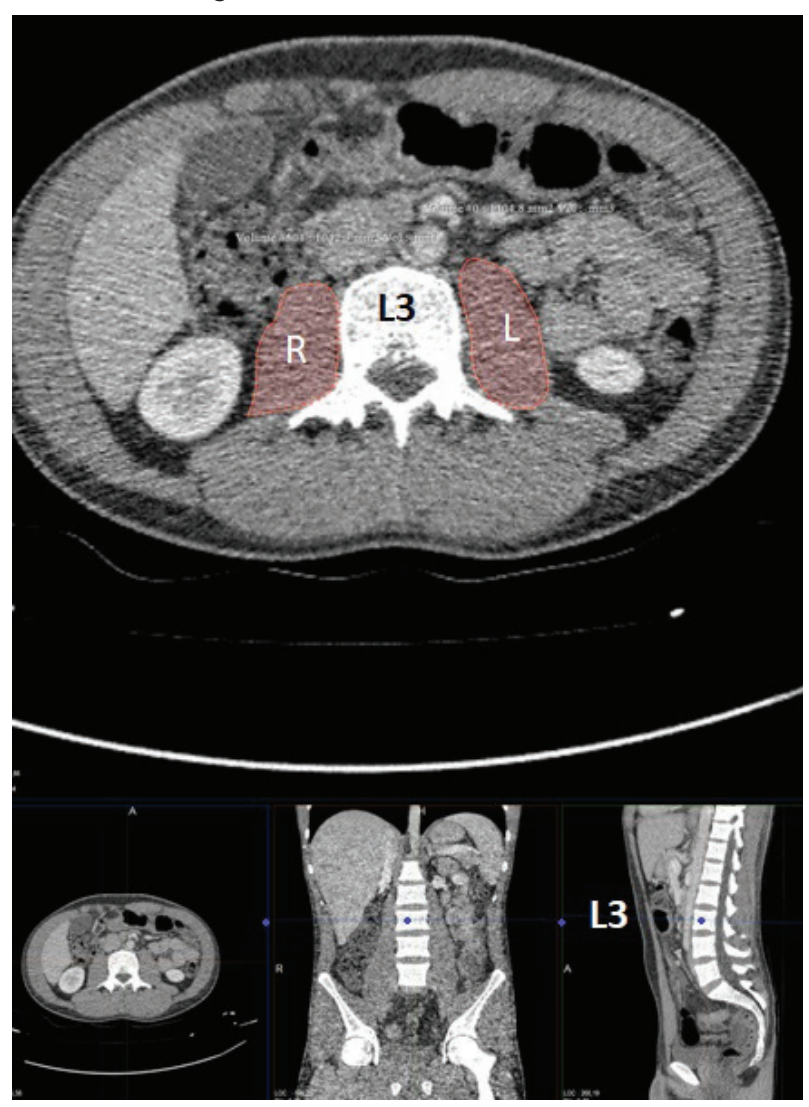

L3: Corpus of the $3^{\text {rd }}$ lumbar vertebra

R: Right psoas muscle

L: Left psoas muscle 
probability plots, and histograms. The PMI and CFS scores were not normally distributed. Median (minimum-maximum) or mean (standard deviation) were used to describe continuous variables, and frequency and percentage were used when defining categorical variables. Student's t-test was used to differentiate the means of two independent groups, and an ANOVA was used to compare the means of more than two separate groups. When differences were detected with an ANOVA, Tukey's test was used as a post hoc test. Chi-square tests were used to evaluate the relationships between categorical variables. The data were non-normally distributed; therefore, Spearman's analysis was used. To interpret the Spearman's rho ( $r$ ) coefficient, we used the following benchmarks: 0-0.20, poor correlation; $0.21-0.40$, fair correlation; $0.41-0.60$, moderate correlation; $0.61-0.80$, substantial/strong correlation; and $0.81-1.0$, near-perfect correlation. To determine the effects of impact factors on survival, the log-rank test, the test statistic in the Kaplan-Meier method, was used. The time to reach a relevant event (death) was measured in months of follow-up. A value of $p$ $<0.05$ was considered statistically significant. SPSS 22 version statistics package program was used to analyze the data.

\section{RESULTS}

The median age of the patients was 64 (37-84) years, and 57 (51.3\%) of the patients were female. $67(60.3 \%)$ of the patients were 65 years or older. The patients' demographic characteristics, laboratory and anthropometric values, and disease-specific findings at diagnosis are summarized in Table 1. Sarcopenia was detected in 46 (41.4\%) of the patients. There was a significant association between sarcopenia and death $(p=0.04)$.

There was a weak correlation between HUAC and PMI ( $r=0.269, p=0.02$ ). Neither HUAC nor PMI correlated with Body Mass Index (BMI). The mean HUAC was $41.44 \pm 8.11$ and $36.98 \pm 7.13$ in patients with sarcopenia and without sarcopenia, respective-
Table 1. Descriptive characteristics of the patients

\begin{tabular}{|l|c|}
\hline & Median (min-max) \\
\hline Psoas Index $\left(\mathrm{mm}^{2} / \mathrm{m}^{2}\right)$ & $424.87(220.88-857.02)$ \\
\hline Female & $366.33(220.89-560.08)$ \\
\hline Male & $585.21(297.16-857.02)$ \\
\hline
\end{tabular}

CFS scores according to sarcopenia status

\begin{tabular}{|c|c|c|}
\hline Sarcopenic $(n=46,41.4 \%)$ & \multicolumn{2}{|c|}{$5(3-8)$} \\
\hline Non-Sarcopenic ( $n=65,58.6 \%)$ & \multicolumn{2}{|c|}{$4(1-7)$} \\
\hline HUAC (HU) & \multicolumn{2}{|c|}{$39.38(24.1-62.42)$} \\
\hline Female & \multicolumn{2}{|c|}{$38.26(24-62)$} \\
\hline Male & \multicolumn{2}{|c|}{$39.64(24-54)$} \\
\hline \multicolumn{3}{|l|}{ Body mass index } \\
\hline Female & \multicolumn{2}{|c|}{$25.00(22.09-34.88)$} \\
\hline Male & \multicolumn{2}{|c|}{$28.68(18.75-45.79)$} \\
\hline Body surface area $\left(\mathrm{m}^{2}\right)$ & \multicolumn{2}{|c|}{$1.84(1.43-2.78)$} \\
\hline & & n (\%) \\
\hline \multirow[t]{3}{*}{ ISS stage } & 1 & $12(10.8)$ \\
\hline & 2 & $69(62.1)$ \\
\hline & 3 & $30(27.1)$ \\
\hline \multicolumn{2}{|l|}{ Death during follow-up } & $33(29.7)$ \\
\hline \multicolumn{2}{|c|}{ Renal failure at the time of diagnosis } & $49(44.1)$ \\
\hline \multicolumn{2}{|c|}{ History of ASCT } & $18(16.2)$ \\
\hline
\end{tabular}

Abbreviations: ASCT: autologous stem cell transplantation, CFS: Clinical Frailty Scale, HUAC: Hounsfield unit average calculation, ISS: International Staging System.

ly $(p=0.017)$. HUAC was not associated with survival.

The median follow-up duration of the patients was 21.7 (1.51-74.97) months. The median overall survival was 42.3 months. Patients without sarcopenia had not yet reached the median overall survival. The mortality risk was significantly higher in patients with sarcopenia than in patients without sarcopenia (log-rank $\mathrm{p}=0.028$, HR: 2.43, 95\% Cl: 1.02-5.77) (Figure 2). The one-year cumulative survival rates were $77 \%$ and $92 \%$ in patients with and without sarcopenia, respectively.

While 32 (28\%) of the patients were robust, 79 (72\%) of the patients were frail. When survival 
curves were examined separately according to CFS category, frailty significantly affected overall survival (HR: 3.28, 95\% Cl: 1.28-8.41, $p=0.01$ ) (Figure 3). There was a fair correlation between CFS score and PMI $(r=-0.464, p<0.001)$.

\section{DISCUSSION}

This was a rare study examining the association between sarcopenia (cross-sectional area by CT) and overall survival in patients with MM. Since particular attention is paid to muscle quality and quantity in the EWGSOP2, we evaluated the PMI and HUAC in patients with newly diagnosed MM. There was a significant relationship between PMI and overall survival in these patients. This study is the first to use population-specific PMI cut-off values in patients with MM. This is also the first study to show the re-

Figure 2. Comparison of overall survival between patients with and without sarcopenia

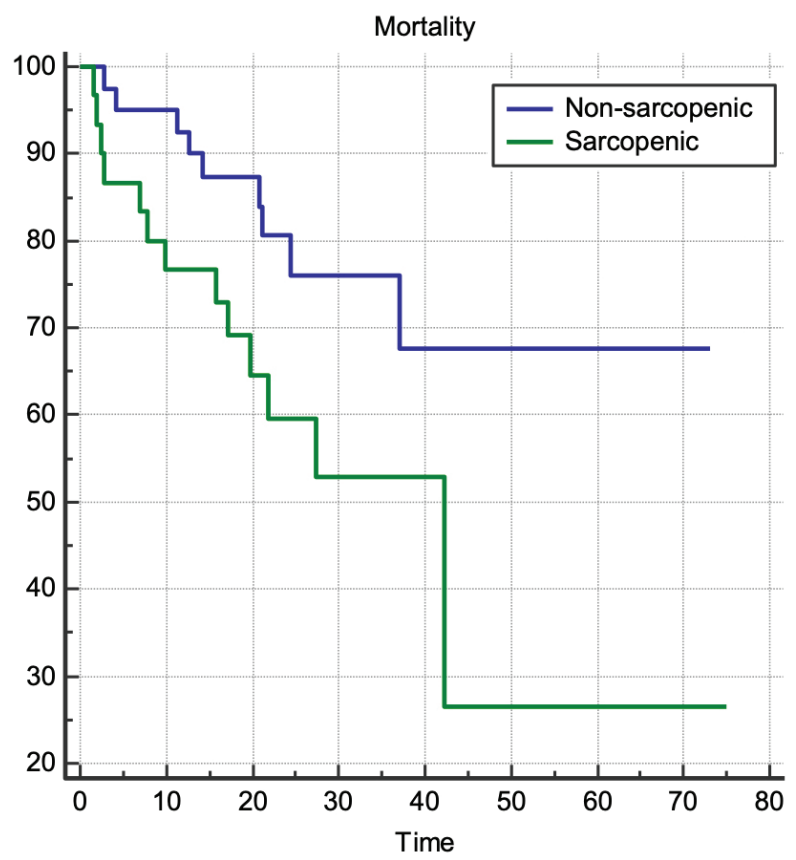

lationship between retrospectively determined CFS and overall survival in patients with MM.

Since $\mathrm{MM}$ is a hematological malignancy that occurs mainly in the elderly population and immobility due to disease-related bone involvement is common in these patients, the risk of sarcopenia is high in these patients. In this study, the prevalence of sarcopenia was $41.4 \%$, which is similar to that observed in previous studies[16].

We found four studies in the literature on the relationship between psoas muscle mass and overall survival in patients with MM[16-18]. Only one of these studies demonstrated that low muscle mass worsened overall survival[18]. However, tertiles were used instead of population-specific cut-offs in that study. Other studies were examined in detail. Takeoka et al. evaluated skeletal muscle indexes[16].

Figure 3. Comparison of overall survival according to retrospective Clinical Frailty Scale scores

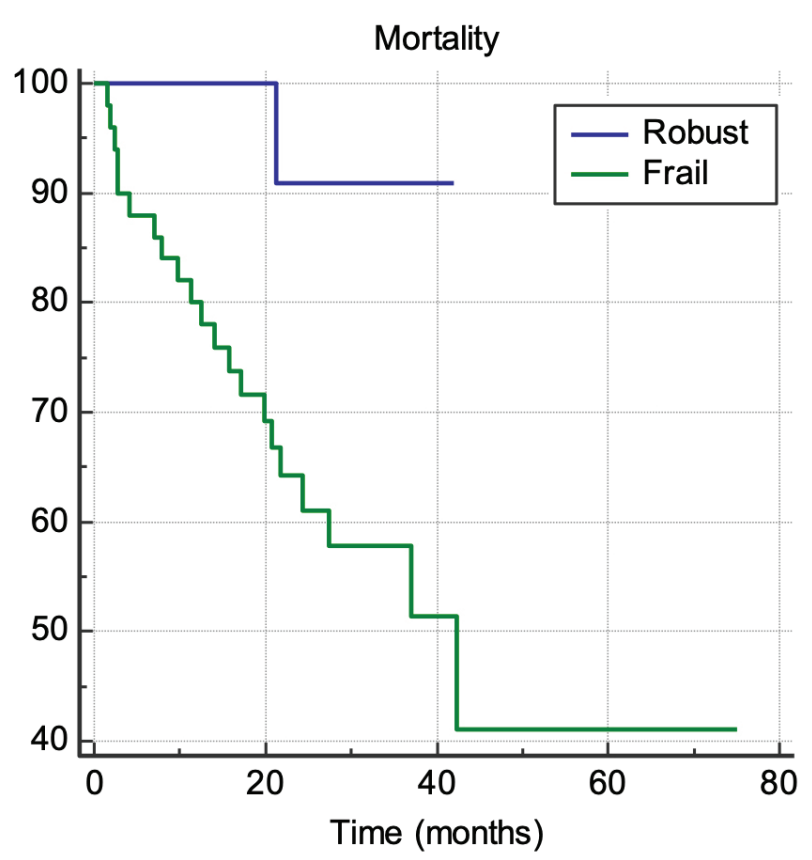


The cut-offs used by Williams et al. were unclear[17]. Bhamidipati et al. used tertiles[19]. The EWGSOP2 recommends the determination of PMI cut-offs according to specific populations. A recent study conducted by Bahat et al. with 601 healthy cases revealed gender-specific PMI cut-offs in the Turkish population[10]. We used these cut-off values in our study. The fact that the cut-off values we used for the PMI were determined in the Turkish population increased the strength of this study.

Frailty is a geriatric syndrome that has been reported to be associated with sarcopenia in the EWGSOP2. Previous studies have shown that frailty is also associated with survival in patients with $M M[13,20]$. However, we could not find any studies in the literature on the effect of retrospective CFS scores on survival outcomes in patients with MM. The CFS has previously been shown to be a valid and reliable tool to measure frailty in the Turkish population[11]. In a recent study by Stille et al., the retrospective use of CFS was reliable in older adults who were hospitalized[12]. We retrospectively determined the CFS scores in our patients and examined the mortality of these patients. As with sarcopenia, frailty influences mortality in these patients. This study was crucial because it revealed that the retrospective CFS score can be used to predict mortality in patients with MM. More studies regarding the use of retrospective CFS scores to predict the prognosis of patients with malignant diseases are needed. On the other hand, there is an increasing number of studies in the literature showing that frailty scores may be important when deciding on autologous stem cell transplantation (ASCT) in patients with MM[21]. Among the ASCT eligibility criteria, PMI and CFS may be parameters that should be emphasized.

The concept of muscle quality is gaining attention as a relatively new concept in the EWGSOP2. The logic is that as adipose tissue in the muscle increases, muscle quality decreases and density changes. Many studies show that muscle density is also associated with poor prognosis in patients with solid tumors[22, 23]. Although a significantly weak correlation between HUAC and muscle mass was shown in this study, no effect on survival was observed. The cut-off values for HUAC remain a mystery, and new studies are needed.

The limitations of this study include its retrospective nature and relatively small sample size. Since most patients did not have genetic results, they could not be included in the analysis. Based on studies showing that the PMI correlates with the total skeletal muscle index, we did not measure the total skeletal muscle index (SMI). Since the information on this subject remains unclear, we could look at the correlation of PMI with total SMI measurement. Since we could not evaluate the patients using physical performance tests, such as the timed up and go test and the 400-m walking test, we could not determine their severity of sarcopenia. Determining the severity of sarcopenia will be important in future studies on this subject because the severity of sarcopenia may be related to complications and the overall survival of patients with $\mathrm{MM}$. We also did not evaluate chemotherapy-related toxicities. Chemotherapy toxicity can be affected by sarcopenia[24, 25]. A prospective study with nutritional support and exercise interventions would be valuable because the effects of treatment on mortality could be investigated.

Sarcopenia is a common finding in patients with MM. Since routine CT scans are performed before treating $\mathrm{MM}$, it is more practical to determine muscle mass with CT in these patients. It is also cheaper and less time-consuming than other methods of evaluating sarcopenia. Sarcopenia can contribute to determining the prognosis as a practical method by using the PMI and can be evaluated together with frailty when arranging treatment. 


\section{REFERENCES}

1. Rajkumar SV, Dimopoulos MA, Palumbo A, et al. International Myeloma Working Group updated criteria for the diagnosis of multiple myeloma. Lancet Oncol. 2014 Nov;15(12):e538-48 (PMID: 25439696).

2. Chindapasirt J. Sarcopenia in Cancer Patients. Asian Pac J Cancer Prev. 2015;16(18):8075-7 (PMID: 26745041).

3. Ethun CG, Bilen MA, Jani AB, Maithel SK, Ogan K, Master VA. Frailty and cancer: Implications for oncology surgery, medical oncology, and radiation oncology. CA Cancer J Clin. 2017 Sep;67(5):362-377 (PMID: 28731537).

4. Salazar AS, Recinos LM, Mian HS, et al. Geriatric Assessment and Frailty Scores Predict Mortality in Myeloma: Systematic Review and Meta-analysis. Clin Lymphoma Myeloma Leuk. 2019 Aug;19(8):488-496. e6 (PMID: 31133527).

5. Gibson DJ, Burden ST, Strauss BJ, Todd C, Lal S. The role of computed tomography in evaluating body composition and the influence of reduced muscle mass on clinical outcome in abdominal malignancy: a systematic review. Eur J Clin Nutr. 2015 Oct;69(10):1079-86 (PMID: 25782424).

6. Prado CM, Lieffers JR, McCargar LJ, et al. Prevalence and clinical implications of sarcopenic obesity in patients with solid tumours of the respiratory and gastrointestinal tracts: a population-based study. Lancet Oncol. 2008 Jul;9(7):629-35 (PMID: 18539529).

7. Cruz-Jentoft AJ, Bahat G, Bauer J, et al. Sarcopenia: revised European consensus on definition and diagnosis. Age Ageing. 2019 Jan 1;48(1):16-31 (PMID: 30312372)

8. Hanaoka M, Yasuno M, Ishiguro M, et al. Morphologic change of the psoas muscle as a surrogate marker of sarcopenia and predictor of complications after colorectal cancer surgery. Int J Colorectal Dis. 2017 Jun;32(6):847-856 (PMID: 28190101).

9. Gu DH, Kim MY, Seo YS, et al. Clinical usefulness of psoas muscle thickness for the diagnosis of sarcopenia in patients with liver cirrhosis. Clin Mol Hepatol. 2018 Sep;24(3):319-330 (PMID: 29706058).

10. Bahat G, Turkmen BO, Aliyev S, Catikkas NM, Bakir $B$, Karan MA. Cut-off values of skeletal muscle index and psoas muscle index at L3 vertebra level by computerized tomography to assess low muscle mass. Clin Nutr. 2021 Jan 16; (PMID: 33516603).
11. Özsürekci C, Balcı C, Kızılarslanoğlu MC, et al. An important problem in an aging country: identifying the frailty via 9 Point Clinical Frailty Scale. Acta Clin Belg. 2020 Jun;75(3):200-204 (PMID: 30919742).

12. Stille K, Temmel N, Hepp J, Herget-Rosenthal S. Validation of the Clinical Frailty Scale for retrospective use in acute care. Eur Geriatr Med. 2020 Dec;11(6):1009-1015 (PMID: 32770462).

13. Facon $T$, Dimopoulos MA, Meuleman $N$, et al. A simplified frailty scale predicts outcomes in transplant-ineligible patients with newly diagnosed multiple myeloma treated in the FIRST (MM-020) trial. Leukemia. 2020 Jan;34(1):224-233 (PMID: 31427722).

14. Sakatoku K, Takeoka Y, Miura A, et al. Combination of Frailty Status and Comorbidity Score Improves the Stratification of Survival in Patients With Myelodysplastic Syndrome Owing to Good Predictive Capability for Infection-related Mortality. Clin Lymphoma Myeloma Leuk. 2019 Dec;19(12):799-805 (PMID: 31648956).

15. Rockwood K, Song X, MacKnight C, et al. A global clinical measure of fitness and frailty in elderly people. Cmaj. 2005 Aug 30;173(5):489-95 (PMID: 16129869).

16. Takeoka Y, Sakatoku K, Miura A, et al. Prognostic Effect of Low Subcutaneous Adipose Tissue on Survival Outcome in Patients With Multiple Myeloma. Clin Lymphoma Myeloma Leuk. 2016 Aug;16(8):434-41 (PMID: 27263047).

17. Williams A, Baruah D, Patel J, et al. Prevalence and significance of sarcopenia in multiple myeloma patients undergoing autologous hematopoietic cell transplantation. Bone Marrow Transplant. 2021 Jan;56(1):225-231 (PMID: 32732941).

18. Zakaria HM, Elibe E, Macki M, et al. Morphometrics predicts overall survival in patients with multiple myeloma spine metastasis: A retrospective cohort study. Surg Neurol Int. 2018;9:172 (PMID: 30210905).

19. Bhamidipati PK, Carson KR, Wildes TM. Psoas Cross-Sectional Area As Radiographic Measure Of Sarcopenia Does Not Predict Overall Survival In Multiple Myeloma. Blood. 2013;122(21):5326-5326).

20. Lee HS, Kim K, Lee JJ, et al. Clinical impact of frailty on treatment outcomes of elderly patients with relapsed and/or refractory multiple myeloma treated with lenalidomide plus dexamethasone. Int J Hematol. 2021 Jan;113(1):81-91 (PMID: 32889695).

21. Belotti A, Ribolla R, Cancelli V, et al. Transplant eligi- 
bility in elderly multiple myeloma patients: Prospective external validation of the international myeloma working group frailty score and comparison with clinical judgment and other comorbidity scores in unselected patients aged 65-75years. Am J Hematol. 2020 Jul;95(7):759-765 (PMID: 32242970).

22. Dohzono S, Sasaoka R, Takamatsu K, Hoshino M, Nakamura $\mathrm{H}$. Prognostic value of paravertebral muscle density in patients with spinal metastases from gastrointestinal cancer. Support Care Cancer. 2019 Apr;27(4):1207-1213 (PMID: 30220027).

23. Cortellini A, Bozzetti F, Palumbo P, et al. Weighing the role of skeletal muscle mass and muscle density in cancer patients receiving PD-1/PD-L1 checkpoint inhibitors: a multicenter real-life study. Sci Rep. 2020 Jan 29;10(1):1456 (PMID: 31996766).

24. Vega MC, Laviano A, Pimentel GD. Sarcopenia and chemotherapy-mediated toxicity. Einstein (Sao Paulo). 2016 Oct-Dec;14(4):580-584 (PMID: 28076611).

25. Bozzetti F. Forcing the vicious circle: sarcopenia increases toxicity, decreases response to chemotherapy and worsens with chemotherapy. Ann Oncol. 2017 Sep 1;28(9):2107-2118 (PMID: 28911059). 